\title{
Creación de micromundos aplicando la teoría de juegos y el diseño orientado a objetos
}

\author{
Clemencia Alava Viteri ${ }^{1}$ \\ Adriana Aguirre Cabrera ${ }^{2}$ \\ Harold Emilio Cabrera Meza ${ }^{3}$ \\ Sixto Enrique Campaña Bastidas ${ }^{4}$ \\ Jorge Aníbal Maya
}

\section{Resumen}

Existen en el mercado varios tipos de video juegos con diferentes características como los juegos de arcade, de disparos, de aventura, de rol, educativos, musicales y otra gran cantidad de escenarios. Cada videojuego tiene un proposito o reto que debe ser superado con el ánimo de obtener un premio. Este tipo de programas tiene la característica peculiar de estar construido por niveles de dificultad, donde el premio varia de acuerdo con el reto superado. Destacar este aspecto en los juegos de video permite diseñar juegos con niveles de aprendizaje que se van superando a medida que los individuos interactúan con los conocimientos que les otorga el juego en cada nivel de aprendizaje o nivel del juego.

Palabras Glave: Micromundos, lógica matemática, pensamiento geométrico, pensamiento lógico.

\footnotetext{
1 Magister Clemencia Alava Viteri: Universidad Nacional Abierta y a Distancia, Decana espejo ECBTI; clemencia.alava@unad. edu.co; Pasto - Colombia.

2 Especialista Adriana Aguirre Cabrera: Universidad Nacional Abierta y a Distancia, Tutora TC ECBTI; carmen.aguirre@unad. edu.co; Pasto - Colombia.

3 Ingeniero Harold Emilio Cabrera Meza; Universidad Nacional Abierta y a Distancia, Tutor TC ECBTI; harold.cabrera@unad.edu.co; Pasto - Colombia.

4 Magister Sixto Enrique Campaña Bastidas. Universidad Nacional Abierta y a Distancia, Docente ECBTI; carmen.aguirre@ unad.edu.co; Pasto - Colombia.

5 Candidato a Doctor Jorge Anibal Maya Universidad Nacional Abierta y a Distancia, Tuto TC ECAPM A; jorge-anibal@unad. edu.co; Pasto - Colombia.
} 
Clemencia Alava Viteri, Adriana Aguirre; Harold Cabrera; Sixto Campaña; Jorge Aníbal Maya

Creación de micromundos aplicando la teoría de juegos y el diseño orientado a objetos, artículo de producción científica

\title{
Creating micro worlds by applying the theory of games and object-oriented design
}

\begin{abstract}
On the market there are various types of video games, which display varied features, such as arcade games, shooting, adventure, role playing, educational, musical and a number of other scenarios. Each video game has a purpose or challenge to be overcome with the purpose of obtaining a prize. Such programs have the unique feature of being built by levels of difficulty in which the aw ard varies according the challenges to overcome. Highlighting this aspect in video games can help design games with learning levels that are overcome as individuals interact with knowledge the game aw ards at each level of learning or level of play.
\end{abstract}

Keywards: Micro worlds, mathematical logic, geometric thinking, logical thinking.

Recibido: 12 Enero de 2011

Aceptado: 27 Mayo de 2011

\section{lntroducción}

En la actualidad los procesos educativos se apoyan en aran medida en las tecnologlas de la información y la comunicacion $(T / C)$. Estos recursos informáticos son variados y de differentes usos, ajustandose a las necesidad de los usuarios. Algunos recursos populares son, por ejemplo: las, redes sociales, los chats, los foros, los wikis, los blogs, los videojuegos, las páainas web como referencia a los mas usados, Entre esa gama de recursos que nos ofrecen las TIC, centraremos la atencion sobre los juegos de computador o videojuegos, tal vez uno de los recursos informaticos mas llamativos por su vistosidad, colorido, musica y accion. La Free Patents Online FPO define que un "videojuego es un programa creado para el entretenimiento en general y basado en la interaccion entre una 0 varias personas y un aparato electronico que hace las veces de puente entre el usuario y el programa". La palabra "video" determina que el programa tiene un gran contenido para ser visual izado y de esto depende en gran medida el interés que se genera hacia la persona que lo utiliza. 
Revista de

investigaciones UNAD

Volumen 10. Número 1. Junio 2011

Construcción de micromundos educativos

Las teorías pedagógicas han creado muchas formas de mejorar el proceso enseñanza-aprendizaje, incluyendo tecnologias informáticas que permiten crear nuevos ambientes lúdicos de aprendizaje para estudiantes y profesores. Sin embargo, es neccesario para los micromundos fundamentar su diseno mediante una metodologia pedagogica, los recursos informaticos y la teoria de juegos T]. Para los dos primeros aspectos, el matemático e investigador Seymour Papert. especial ista en educación eintel igencia artificial, acuño el termino "micromundo" a fines de los años 70. El término designa toda simulación donde los individuos pueden "vivir", realizar experimentos, verificar estrategias y elaborar una mejor comprension de los aspectos del mundo real que aparecen retratados en el micromundo. En esencia, Seymour Papert integra el proceso ensenanzaaprendizaje mediante un ambiente que simula el mundo real, usando medios informaticos donde el individuo puede llegar a comprender de manera simple los conceptos que se quieren dar a conocer mediante la construccion de objetos y el diseño de programas informáticos. Esta manera de aprender haciendo tiene su fundamento en lo que Seymour Papert definió como construccionismo. Esta teoría plantea que "los sujetos, al estar activos mientras aprenden, construyen tambien suș propias estructuras de conocimiento de manera paral ela a la construccion de objetos. Tambien afirma que los sujetos aprenderan mejor cuando construyan objetos que les interesen personal mente, al tiempo que los objetos construidos ofrecen la posibilidad de hacer mas concretos y pal pables los conceptos abstractos o teóricos y por tanto, los hace mas fácilmente comprensibles." Esta teoría, fundamentada en las teorías constructivistas de Piaget, manifiesta que para que se produzca el aprendizaje el individuo debe construir y reconstruir el conocimiento a traves de la accion.

L os micromundos son herramientas con un fundamento pedagógico conștructivista que simulan el mundo real en un computador, permitiendo a los individuos tomar decisiones, analizar casos, cometer errores y dar sol uciones a un problema determinado, todo esto en un ambiente computacional seguro que les permite experimentar en diferentes escenarios sus conocimientos. Proveen al individuo de una interfaz atractiva y facil de manipular que le permite util lizar los modelos de al guna teoria para experimentar y aprender con ellos. Dicha experimentación y los resultados generados ocașionan la formulación y reformulación de sus modelos mentales y en consecuencia representar un conocimiento cada vez mas cercano al objeto de estudio.

La manera más particular de mostrar un escenario que permita la interacción dinamica del individuo entre el conocimiento y su experiencia cognoscitiva es el juego de video que reune las condiciones básicas del micromundo. Es necesario involucrar la teoría de juegos en la construccion del micromundo dado que el tratamiento que se debe dar al juego se debe fundamentar en los preceptos que esta teoría aporta. John Von Neumann explica en la teoría de juegos que este tipo 
Clemencia Alava Viteri, Adriana Aguirre; Harold Cabrera; Sixto Campaña; Jorge Aníbal M aya

Creación de micromundos aplicando la teoría de juegos y el diseño orientado a objetos, artículo de producción científica

de análisis matemático fue diseñado para realizar la predicción de un resul tado que se puede dar entre dos o mas individuos por la disputa de un bien 0 un servicio. Neumann demuestra que en un juego existen probabilidades de que un individuo gane todo, que pierda o que comparta un premio. De acuerdo con esta premisa inicial sobre el esquema general del juego, se tiene el punto de partida para destacar que en un juego un individuo puede ganar o perder.

En el aprendizaje, perder o ganar se centra en la manera como un individuo puede adquirir un conocimiento y como el individuo se apropia de este nuevo saber para aplicarlo en su quehacer diario o de lo contrario, no obtener de ese conocimiento ningún beneficio, es decir "ganar" nuevos conocimientos 0 "perder" oportuni dades de aprender al go nuevo. La aplicación de este concepto se centra en la construccion de las reglas y escenarios del micromundo, aplicar la teoria de juegos en esta construccion dá al micromundo vali dez en su diseno y modularidad para ser aplicado en cualquier area del conocimiento.

Micromundos utilizando la teoría de juegos

La combinación de las herramientas que provee el micromundo y la teoría de juegos resul ta interesante, ya que por una parte se tiene al micromundo como el elemento didactico computacional que recrea un ambiente simulado de la realidad en un computador y la teoria de juegos como las reglas a seguir en el desarrollo del juego. Los conceptos basicos de la teoría de juegos se explican como sigue:

El rol del jugador: Identifica la conducta de cada jugador de manera que pueda saberse de que forma probable se comportará. Con esto se determina cual es su rol dentro del juego y como busca su maximo bienestar, De esta forma se estipulan las funciones que persigue el jugador dentro del juego sin llegar a excederlas.

La finalidad (objetivo) del juego: En este aparte se diseña el quión del. juego, donde se establecen los objetivos a lograr dentro del juego: los beneficios que se pueden obtener al realizar una tarea, resolyer un acertijo, debatír con otros adversarios o realizar exitosamente una mision. La utilidad se entiende como el premio o castigo que se obtiene dentro del juego, lo que se gana o lo que se pierde Para el micromundo educativo, el objetivo del juego es que los jugadores (estudiantes o profesores) inicien el proceso de aprendizaje a partir de los elementos educativos que les presente el juego, logrando un acercamiento al nuevo conocimiento que se quiere impartir. Por ejemplo, la geometria se puede enseñar recreando un micromundo educativo donde se muestren de manera simulada las propiedades de las figuras geométricas. Harold Cabrera expone que los micromundos ap licados a la geometría son una buena forma de explicar conceptos abstractos, apoyada en una base teorica que explique de manera didactica la enseñanza de la geometría para ser recreada en un micromundo. 
Revista de

investigaciones UNAD

Volumen 10. Número 1. Junio 2011

La solución dęl juego: La solución de un juego es la combinación de ganancias o perdidas que da el jueg.o. Para los micromundos educativos existirá el concepto de ganancia siempre porque, como se explica en la finalidad del juego, el micromundo enseña nuevos conocimientos. "Ganar" implica que el jugador (estudiante o profesor) obtenga un concepto claro y explicado de un conocimiento.

Orden de los movimientos en el juego: Un juego puede ser de movimientos simultáneos o de movimientos secuenciales. El micromundo al recrear un escenario del mundo real permite que jugador o jugadores compartan su experiencia permitiendo el diálogo y el intercambio de ideas.

Con los el ementos de construcción del juego definidos por la teoría de juegos se involucran en la construccion de micromundo inicial mente en determinar la finalidad del juego que consisten en recrear los escenarios donde se desenvuelve la trama o historia del juego como por ejemplo un parque, una casa, una grania o un lugar que no sea desconocido para el jugador. En este escenario se presentan los objetos, las personas o aliados que proveen al jugador de la informacion necesaria que le permite superar el reto. En el micromundo educativo esta información consiste en mostrar conceptos o teorías propias de un saber. Por ejemplo, Harold Cabrera recrea mediante un juego de rol el escenario donde muestra al jugador interactuando con otros personajes que centran su atencion en el estudio de la geometria, logrando con esto definir de manera clara el objetivo del juego.

Construido en el micromundo educativo el escenario de juego y de acuerdo con la teoria de juego, se hace necesario determinar el rol o el papel que desempeña el jugador dentro del juego. Este debe buscar, indagar e interactuar con el escenario y personajes diseñados dentro del juego. La tarea del jugador es la de explorar el micromundo en todos sus niveles, lograr resolver cada situacion del juego segun la experiencia adquirida en cada nivel. Es entonces el jugador (profesor o estudiante) guien asume el rol del personaje principal y asume como propio lo que enseña el micromundo educativo.

La solución es diversa para cada juego, pero en los micromundos educativos la solución de un juego radica en como el jugador soluciona los problemas planteados de acuerdo con la experiencia adquirida en el micromundo, por lo cual la forma de resolver un juego varia segun se contextualice lo aprendido.

En el micromundo educativo se dan siempre las indicaciones correctas para que el jugador explore las soluciones, corrigiendo las erroneas y fortaleciendo las correctas.

Los juegos de rol aplicados para los micromundos son una forma de recrear escenarios aj ustados a la realidad que se quiere simular, Permiten, libertad: de movimientos, de premios, de escenarios, de castigos y de información, ya que se 
Clemencia Alava Viteri, Adriana Aguirre; Harold Cabrera; Sixto Campaña; Jorge Aníbal Maya

Creación de micromundos aplicando la teoría de juegos y el diseño orientado a objetos, artículo de producción científica

puede presentar de manera concurrente al jugador incrementado su experiencia y aprehension en el conocimiento que se quiere ensenar.

Seguimiento al jugador del micromundo

La teoría de juegos tiene diferente maneras de verificar si un jugador ha logrado o no el objetivo de un juego. El seguimi ento adecuado que se aplica a los jugadores de un micromundo educativo es el de los árboles de resultados sucesivos. Este tipo de evaluacion se util liza en juegos que implican secuencias de movimientos. A ugusto Rufasto explica que un arbol de resul tados sucesivos define un punto de partida dentro del juego para el jugador. A partir del inicio del juego o del primer movimiento del jugador, cada escenario extiende ramas que representan los diferentes movimientos que puede realizar el jugador que inicia la competencia. Los diferentes movimientos o ramas definiran igual número de resultados o pagos (ganancia o pérdida), gue pueden servir como punto de partida para nuevas decisiones del jugador. El proceso se repite hasta completar el número de movimientos que el jugador pueda real izar. Las puntas de las ramas del ultimo movimiento contienen la descripcion de los posibles resul tados del juego. Para los micromundos educativos esto se interpreta como la forma en que el jugador (estudiante o profesor) logro llegar al final del juego solucionado los interrogantes que evaluán al guna competencia educativa suya.

Aplicación del enfoque orientado a objetos en la creación de micromundos

Analizados los preceptos teóricos que permiten sustentar el diseño de micromundos mediante la aplicacion de la teoria de juegos queda demostrar que, en el plano de la creacion de software, los micromundos son de facil desarrollo en cualquier plataforma informática. Para su construccion los micromundos deben ser di senados desde cero porque cada micromundo ensena nuevos saberes de forma diferente, Para ello se necesita diseñar el "plano" del software donde se plasmarán las caracteristicas del software a crear.

Utilizando el enfoque orientado a objetos $(0,0$.$) se puede hacer representación$ del mundo que se desea modelar en términos de los objetos que posee segun se determinan en la teoria de juegos. Cada uno de los elemento tiene sus propias caracteristicas que lo identifican y que definen su comportamiento dentro del juego. Estos aspectos pueden formal izarse con este enfogue ya que se pueden tomar las caracteristicas especificas de cada elemento sin dar lugar a repetir elementos. Con base en las caracteristicas y comportamiento del objeto se pueden definir variantes que deben cumplirse, permitiendo así verificar que el objeto funciona como se quiere. Con el modelado unificado de modelado UM L se real iza el diseño de manera teórica o en papel del software que se desea realizar con el enfoque orientado a objetos. 
Revista de investigaciones UNAD

Volumen 10. Número 1. Junio 2011

Galvis hace una propuesta formal sobre la construcción de un micromundo educativo que inicia con el análisis. El objetivo de esta etapa es determinar el contexto donde se va a crear la aplicacion. En esta fase se tiene en cuenta lo definido en el objetivo del juego y derivar de alli los requerimientos que debera atender la solucion interactiva, como complemento a otras soluciones basadas en uso de otros medios (personales, impresos, audio-visuales, experienciales), teniendo claro el rol de cada uno de los medios educativos seleccionados y la viabilidad de usarlos.

De acuerdo con Galvis en esta etapa se establece como mínimo la siguiente informacion:

- Características de la población objetivo: edad (física y mental), sexo, características fișicas y mentales (și son relevantes), experiencias previas, expectativas, actitudes, aptitudes, intereses o motivadores por aprender.

- Conducta de entrada y campo vital: nivel escolar, desarrollo mental, físico o psicológico, entorno familiar y escolar.

\begin{abstract}
- Una vez identificcado el problema se deben establecer las bases para resolverlo. Principios pedagógicos y didácticos aplicables. En esta fase se debe analizar cómo se hallevado a cabo el proceso de enseñanzaaprendizaje para establ ecer como debe enfocarse el ambiente, que factores tomar en cuenta, que objetivos debe cumplir.
\end{abstract}

Galvis propone que al diseñar el ambiente donde se desarrollan las acciones se deben definir claramente los elementos, que se determinaron como necesarios en el micromundo educativo, según se analizo en el apartado "micromundos utilizando la teoría de juegos" de este documento, y aquellos deseables que convenga para el caso como pueden ser: argumento e historia, variables compensatorias, variables de control, variables de resultado mundo ( escenarios, retos implicitos / explicitos), personajes y roles, objetos / herramientas, zonas de comunicacion, mecanismos de comunicación usuarioaplicacion, ambientacion / caracterizacion. Estos elementos deseables son los requeridos y propuestos por la teoría de juegos.

Con el enfoque 0,0 . se definen las clases que identifican a cada uno de estos elementos. Algunas de estas clases seran la base sobre la cual se puede extender el micromundo. Al realizar el modelaje del, mundo se deben definir las relaciones existentes entre estas clases. Esta relación se mi ra de manera mas conveniente en la figura 1. 
Clemencia Alava Viteri, Adriana Aguirre; Harold Cabrera; Sixto Campaña; Jorge Aníbal Maya

Creación de micromundos aplicando la teoría de juegos y el diseño orientado a objetos, artículo de producción científica

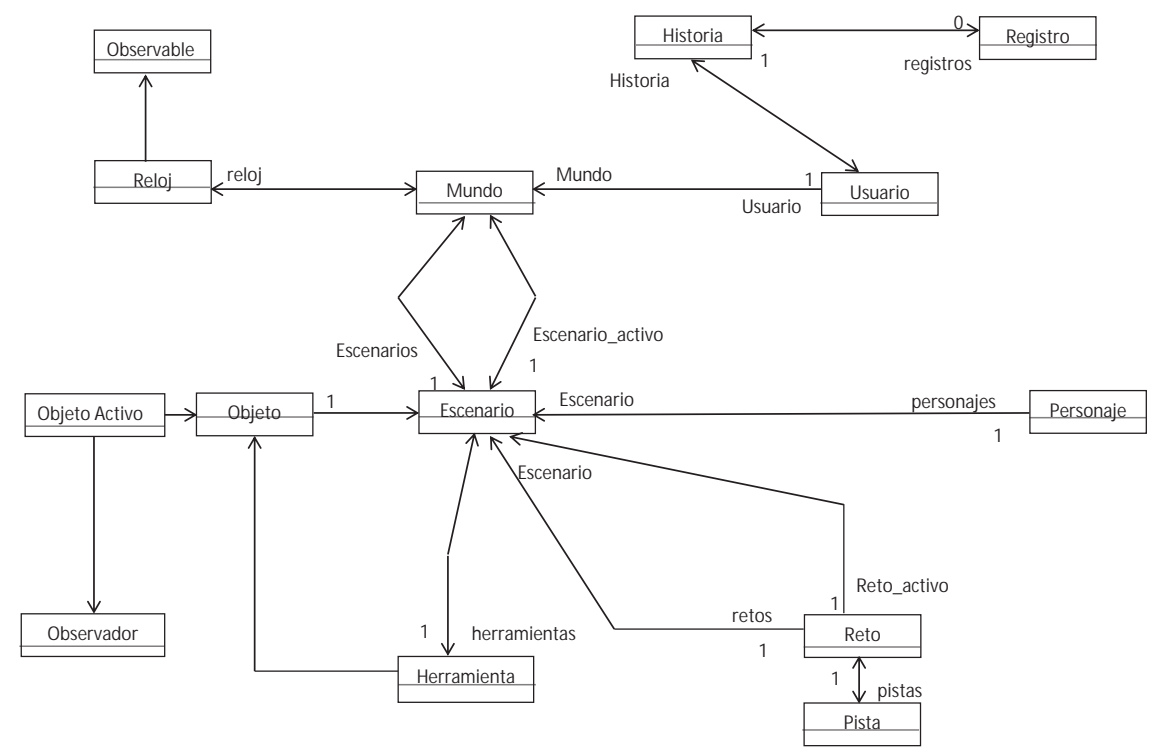

Figura 1. Diagrama de clases del micromundo

Además se debe definir qué cosas puede hacer el usuario en el mundo. En términos de UML se refiere a los casos de uso en el mundo. Los casos de uso se identifican al establecer los requerimientos de información que debe satisfacer la aplicación. Los casos de uso pueden extenderse de acuerdo con las necesidades, del problema. Cada caso de uso se especifica usando djagramas de interaccion que permitan ver los objetos que estan involucrados asi como la secuencia de mensajes entre ellos.



Figura 2. Diagrama de casos de uso del micromundo 
Revista de

investigaciones UNAD

Volumen 10. Número 1. Junio 2011

Con los micromundos educativos es vital despertar motivación intrínseca proponiendo ambientes o situaciones que sean interesantes, que despierten curiosidad, que inviten al usuario a indagar a traves de la experimentacion con el micromundo. Hay que mantener motivados a los usuarios para que el trabaio que se tenga con la aplicacion sea efectivo y de provecho. El micromundo debe ser novedoso y buscar sorprender al usuario, darl e nuevas oportunidades de accion y plantear nuevos retos. Esto aumenta la curiosidad de los usuarios y los mantiene atentos al desarrollo del trabajo con la aplicacion. Complementariamente, se deben plantear retos que mantengan al erta a usuario en busca de pistas para resolverlos y con un nivel de complejidad apropiado.

\section{Evaluación en el micromundo}

Para los micromundos es indispensable definir cómo se va a realizar el seguimiento al jugador (profesor o estudiante), en cuanto a la forma como el jugador ha logrado el objetivo del juego. El análisis que se puede hacer a la forma de "evaluar" esta en como el jugador adquiere las habilidades que le permiten superar los escenarios que tiene disenado el micromundo, De acuerdo con esto, alcanzar cada nivel del juego implica que se han adquirido ciertas habilidades que se premian de acuerdo con el escenario resuelto. En cada escenario se tienen en cuenta los aspectos cognitivos que el jugador ha desarrollado y la forma como los ha aplicado en el juego bajo el criterio del aprendizaje basado en problemas. Estos indicadores de logro deben llevarse como registro en la historia que el individuo ha desarrollado en el transcurso del juego. Este seguimiento se realiza aplicando, lo descrito en el "Seguimiento al jugador del micromundo" descrito en este artículo.

En función del momento de evaluación existe la evaluación formativa que permite retroalimentar al individuo sobre situaciones del juego que necesitan explicacion, permitiendo descubrir o practicar nuevos conceptos que posteriormente se transfieren a los conocimientos del jugador que le posibilitan afianzar sus destrezas, conceptos o habilidades fortaleciendo el proceso ensenanza - aprendizaje.

Retroalimentación y refuerzo

De acuerdo con el enfoque del micromundo desarrollado con teoría de juegos, el aprendizaje se realiza por descubrimiento (enfogue heurístico). La retroinformacion se traduce en mostrar en el micromundo el efecto de lo que hizo el jugador, independientemente de si es correcto o no, para que este sea quien analice lo que ha pasado y tome decisiones al respecto. El micromundo debe en todo momento realizar las aclaraciones necesarias sobre los resultados del juego, sean acertados o erroneos, para que el jugador puede tomar las decisiones necesarias de acuerdo con la retroalimentacion del juego y la interpretación que el jugador obtenga de contrastar sus conocimiento con los 
Clemencia Alava Viteri, Adriana Aguirre; Harold Cabrera; Sixto Campaña; Jorge Aníbal Maya

Creación de micromundos aplicando la teoría de juegos y el diseño orientado a objetos, artículo de producción científica

que el micromundo le otorgue en la situación que se, plante en el escenario, Es importante que exista retroalimentacion y explicación detal lada de por qué se da un situacion equivocada en la toma de decisiones dentro del juego. Esto con el fin de reforzar los conceptos adquiridos en el micromundo.

\section{Conclusiones}

- La teoría de juegos proporciona elementos básicos para la construcción conceptual de juegos. Esta teoria muestra y explica adecuadamente la forma de conjugar el objetivo del juego y el rol que asume un jugador dentro del juego en la solucion del reto que se plantea. Esta base teorica permite un diseño adecuado de los micromundos educativos.

- Los micromundos son herramientas tecnológicas novedosas que permiten recrear mundos real es en ambientes computacional es. El diseño del micromundo, ademas de brindar un ambiente grafico llamativo para los usuarios, tiene inmersa una metodologia pedagogica que permite orientar el aprendizaje y reforzar conocimientos. L a base constructivista del micromundo asegura, que el individuo aprenda a su ritmo, con sus conocimientos y su experiencia.

- Ligar la teoría de juegos y el diseño conceptual del micromundo permite generar metodol ogias que aseguran los di senos de software educativo que pueden apoyar el proceso enseñanza - aprendizaje.

- El diseño orientado a objetos permite plasmar en una realidad lo que la teoría de juegos y los micromundos desean presentar a los individuos. El desarrollo de software educativo tiene solidez cuando se aplican metodologlas de diseño novedosas y actuales que generan modelos universales aplicables a los programas que desean enseñar y entretener a la vez.

\section{Referencias bibliográficas}

Augusto Rufasto, Manual de Teoría de J uegos, 2003, 2004, Lima Perú.

Barrows, H. S. (1986). A Taxonomy of problem-based learning methods, en Medical Education, 20/6, 481-486.

Constructionism vs Instructionism - Discurso de Papert conferencia de educadores en Japón, años 1980. 


\section{Revista de investigaciones UNAD \\ Volumen 10. Número 1. Junio 2011}

Cabrera M eza Harold Emilio, Congreso Internacional Virtual Educa 2011, M exico D.F, I Congreso Internacional de Ingenieria, Trujillo, Perú

Galvis, A. H (1.997). M icromundos lúdicos interactivos: Aspectos críticos en su diseño y desarrollo. Informática Educativa, 10 (2), pp. 191-204.

Galvis, A. H (1992). Ingeniería de Software Educativo. Santafé de Bogotá: Ediciones Uniandes.Revista Electrónica "Actualidades Investigativas en Educación" Volumen 4, Número 1, Año 2004

Senge, P. ( 1994) La Quinta Disciplina. Editorial Granica.

Sito web Sobre proyecto SCRATCH http://www.eduteka.org/modulos/9/289/910/1 Definición de pensamiento lógico. [Consultada el 12 de Julio de 2009.] Disponible en: http://definicion.de/pensamiento-logico/

Serrano, José. Primer Congreso Internacional Logico-Matematico en educación infantil [Consultada el 1 de diciembre de 2009.] Disponible en: http://www.waece. org/cdlogicomatematicas/index.php Labinowicz Ed. (1980), Introducción a Piaget. Editorial Fondo Educativo Interamericano, México, [Consultada el 1 de diciembre de 2009.] Disponible en: http://www.uma.edu.ve/carreras/educacion/programas/ cuarto/preescolar/desarrollo_logico_matematico.pdf

Valverde Naranjo, Luis Guillermo MicroM undos Ex y su Integración al ambiente de aprendizaje Enciclopedia, Director Académico América Latina, LCSI.

Von Neumann y M orgenstern; The Theory of Games and Economic Behaviour (1944). [2] 